adrenoleukodystrophy and gangliosidosis. Organomegaly is present in Gaucher's disease, Niemann-Pick disease and mucopolysaccharidosis. Macrocephaly can occur with Alexander's disease and metachromatic leukodystrophy. Special studies to be considered in young adults with idiopathic dementia include: 1) very long-chain fatty acids (adrenoleukodystrophy); 2) mucopolysaccharide screen; 3) arylsulfatase A (metachromatic leukodystrophy); 4) bone marrow for foam cells (NiemannPick disease); 5) copper and ceruloplasmin (Wilson's disease). (Coker S.B. Diagnosis of childhood neurogenerative disorders presenting as dementia in adults. Neurology June 1991; $\underline{41: 794-798) .}$

COMMENT. This review should be of value to the adult neurologist specializing in Alzheimer's disease and other causes of dementia. A knowledge of childhood neurodegenerative disorders would be important in the differential diagnosis of adult dementia.

\title{
ADRENOLEUKODYSTROPHY: CHILDHOOD AND ADULT FORMS COMPARED
}

Saturated, very long-chain fatty acids in erythrocyte membranes, blood plasma, and mononuclear cells were studied in 4 patients with childhoodadolescent adrenoleukodystrophy, 4 patients with adult adrenoleukodystrophy and 19 normal control subjects in the Department of Neurology, National Chikugo Hospital and the Neurological Institute, Kyusu University, Fukuoka, Japan. The ratios of C26:0 to C22:0 in patients were significantly higher than those of normal control subjects. Ratios of C26:0 to C22:0 in mononuclear cells were significantly higher in patients with childhood-adolescent adrenoleukodystrophy than in patients with adult adrenoleukodystrophy, whereas no significant difference in the ratios in erythrocyte membranes and blood plasma were noted. The results suggest that there is a correlation between phenotype and ratio of $\mathrm{C} 26: 0$ to $\mathrm{C} 22: 0$ within mononuclear cells in patients with adrenoleukodystrophy. (Antoku Y. et al. Adrenoleukodystrophy: a correlation between saturated very long-chain fatty acids in mononuclear cells and phenotype (Ann Neurol July 1991; 30: $101-103$ ).

COMMENT. Adrenoleukodystrophy is an X-linked recessive disorder characterized by adrenal insufficiency and demyelination of the CNS along with accumulation of saturated very long-chain fatty acids in tissues. Adrenoleukodystrophy has a wide phenotypical variation including childhood adolescent forms, adrenomyeloneuropathy with adult onset and adult rare forms. Variable phenotypes in a family kindred have been described. Marsh W.W. and Hurst D.L. reported a 20 month-old male who presented with a sudden onset of status epilepticus and cortical blindness; the patient died before his affected 5 year-old brother exhibited any features of ALD (Pediatr Neurol Jan-Feb 1991; 7:50-52). 